PROCEEDINGS OF THE AMERICAN MATHEMATICAL SOCIETY

Volume 126, Number 7, July 1998, Pages 1923-1930

S 0002-9939(98)04766-

\title{
DISCRIMINANTS OF CONVEX CURVES ARE HOMEOMORPHIC
}

\author{
B. SHAPIRO
}

(Communicated by Christopher Croke)

\begin{abstract}
For a given real generic curve $\gamma: S^{1} \rightarrow \mathbb{P}^{n}$ let $D_{\gamma}$ denote the ruled hypersurface in $\mathbb{P}^{n}$ consisting of all osculating subspaces to $\gamma$ of codimension 2 . In this note we show that for any two convex real projective curves $\gamma_{1}: S^{1} \rightarrow$ $\mathbb{P}^{n}$ and $\gamma_{2}: S^{1} \rightarrow \mathbb{P}^{n}$ the pairs $\left(\mathbb{P}^{n}, D_{\gamma_{1}}\right)$ and $\left(\mathbb{P}^{n}, D_{\gamma_{2}}\right)$ are homeomorphic.
\end{abstract}

\section{§0. Preliminaries and Results}

Definition. A smooth curve $\gamma: S^{1} \rightarrow \mathbb{P}^{n}$ is called nondegenerate or locally convex if the local multiplicity of its intersection with any hyperplane does not exceed $n$, i.e. in local terms $\gamma^{\prime}(t), \ldots, \gamma^{(n)}(t)$ are linearly independent at every $t$ or its osculating complete flag is well-defined at every point. A curve $\gamma: S^{1} \rightarrow \mathbb{P}^{n}$ is called convex if the total multiplicity of its intersection with any hyperplane does not exceed $n$.

The set $\mathfrak{C o n}_{n}$ of all convex curves in $\mathbb{P}^{n}$ forms 1 connected component of the space $\mathfrak{N D}_{n}$ of all nondegenerate curves if $n$ is even and 2 connected components (since the osculating frame orients $\mathbb{P}^{2 k+1}$ ) if $n=2 k+1$; see [MSh]. Different results about convex curves show that they have the most simple properties among all curves. In this paper we prove one more result of the same nature.

Definition. A curve $\gamma: S^{1} \rightarrow \mathbb{P}^{n}$ is called generic if at every point $\gamma(t), t \in S^{1}$, one has a well-defined osculating subspace of codimension 2, i.e. in local terms $\gamma^{\prime}(t), \ldots, \gamma^{(n-1)}(t)$ are linearly independent at every $t$.

Note that any smooth curve $\gamma: S^{1} \rightarrow \mathbb{P}^{n}$ can be made generic by a small smooth deformation of the map. The space $\mathfrak{N D}_{n}$ of all nondegenerate curves is enclosed in the space $\mathfrak{G E N} \mathfrak{N}_{n}$ of all generic curves and consists of several connected components. (The number of connected components in $\mathfrak{N D}_{n}$ equals 10 for odd $n \geqslant 3$ and equals 3 for even $n \geqslant 2$; see [MSh].)

Definition. Given a generic $\gamma: S^{1} \rightarrow \mathbb{P}^{n}$, we define its standard discriminant $D_{\gamma} \subset \mathbb{P}^{n}$ to be the hypersurface consisting of all codimension 2 osculating subspaces to $\gamma$.

In many cases (algebraic, analytic etc) the assumption of genericity in the definition of discriminant can be omitted.

The following proposition answers the question posed by V.Arnold in [Ar2], p.37.

Received by the editors December 17, 1996.

1991 Mathematics Subject Classification. Primary 14H50.

Key words and phrases. Convex curves, discriminants.

(C)1998 American Mathematical Society 
Main proposition. a) For any 2 convex curves $\gamma_{1}: S^{1} \rightarrow \mathbb{P}^{n}$ and $\gamma_{2}: S^{1} \rightarrow \mathbb{P}^{n}$ the pairs $\left(\mathbb{P}^{n}, D_{\gamma_{1}}\right)$ and $\left(\mathbb{P}^{n}, D_{\gamma_{2}}\right)$ are homeomorphic.

b) For any convex curve $\gamma$ the complement $\mathbb{P}^{n} \backslash D_{\gamma}$ consists of $\left[\frac{n}{2}\right]+1$ components. All components are contractible to $S^{1}$ for $n$ even, and all but one are contractible to $S^{1}$ for $n$ odd. The remaining component is a cell.

Now we want to place this result into a more general context of associated discriminants in the spaces of (in)complete flags.

Notation. Let $F_{n+1}$ denote the space of all complete flags in $\mathbb{P}^{n}$ (or, equivalently, in $\left.\mathbb{R}^{n+1}\right)$. Given a nondegenerate curve $\gamma: S^{1} \rightarrow \mathbb{P}^{n}$, one can consider its associated curve $\tilde{\gamma}: S^{1} \rightarrow F_{n+1}$, where $\tilde{\gamma}(t)$ is the complete osculating flag to $\gamma$ at $\gamma(t)$. Note that any associated curve $\tilde{\gamma}: S^{1} \rightarrow F_{n+1}$ is tangent to the special distribution of $n$-dimensional cones in $F_{n+1}$, and any integral curve of this distribution is the associated curve of some nondegenerate projective curve; see e.g. [Sh1].

Given a complete flag $f \in F_{n+1}$ and some space $\mathfrak{G}=S L_{n+1} / P$ of (in)complete flags, where $P$ is some parabolic subgroup, one gets the Schubert cell decomposition $\mathfrak{S c h}_{f}$ of $\mathfrak{G}$ as follows. Each cell of $\mathfrak{S c h}_{f}$ consists of all flags in $\mathfrak{G}$ subspaces of which have a given set of dimensions of intersections with the subspaces of $f$. Let $\mathfrak{O}_{f}$ denote the union of all cells in $\mathfrak{S c h}_{f}$ which have codimension at least 2. (Obviously, $\operatorname{codim} \mathfrak{O}_{f}=2$.)

Examples. 1) If $\mathfrak{G}$ equals $\mathbb{P}^{n}$ then $\mathfrak{O}_{f}$ is the subspace of $f$ of codimension 2.

2) If $\mathfrak{G}=F_{3}$ then $\mathfrak{O}_{f}$ consists of 2 copies of $\mathbb{P}^{1}$ intersecting at $f$. The first $\mathbb{P}^{1}$ is the set of flags on $\mathbb{P}^{2}$ with the same point as that of $f$, and the second $\mathbb{P}^{1}$ consists of all flags with the same line as that of $f$.

Definition. For a given curve $c: S^{1} \rightarrow F_{n+1}$ and a space $\mathfrak{G}=S L_{n+1} / P$ of (in)complete flags we define its $\mathfrak{G}$-discriminant $\mathfrak{G} \mathfrak{D}_{c}$ to be the union $\bigcup_{t \in S^{1}} \mathfrak{O}_{c(t)} \subset$ $\mathfrak{G}$. (If $c$ is not a constant map then $\mathfrak{G} \mathfrak{D}_{c}$ is a hypersurface in $\mathfrak{G}$.)

Note that the standard discriminant $D_{\gamma}$ of a nondegenerate curve $\gamma: S^{1} \rightarrow \mathbb{P}^{n}$ can be considered as the $\mathfrak{G}$-discriminant for $\mathfrak{G}=\mathbb{P}^{n}$.

Definition. Two nondegenerate curves $\gamma_{1}: S^{1} \rightarrow \mathbb{P}^{n}$ and $\gamma_{2}: S^{1} \rightarrow \mathbb{P}^{n}$ are called $\mathfrak{G}$-equivalent if the pairs $\left(\mathfrak{G}, \mathfrak{G} \mathfrak{D}_{\tilde{\gamma}_{1}}\right)$ and $\left(\mathfrak{G}, \mathfrak{G} \mathfrak{D}_{\tilde{\gamma}_{2}}\right)$ are homeomorphic. (Recall that $\tilde{\gamma}$ denotes the associated curve of $\gamma$.)

Remark. The notion of $\mathfrak{G}$-equivalence of nondegenerate curves is intrinsically related with the qualitative theory of linear ODE, since each nondegenerate curve in $\mathbb{P}^{n}$ can be represented as the projectivization of the fundamental solution of some linear ODE of order $n+1$; see [Sh2]. The problem of enumeration of $\mathfrak{G}$-equivalent generic curves is apparently a very interesting and difficult question even for $n=2$.

The following conjecture is formulated in [Sh2].

Conjecture. Any 2 convex curves $\gamma_{1}: S^{1} \rightarrow \mathbb{P}^{n}$ and $\gamma_{2}: S^{1} \rightarrow \mathbb{P}^{n}$ are $\mathfrak{G}$-equivalent for any $\mathfrak{G}=S L_{n+1} / P$.

Note that it suffices to prove this conjecture in the case of the space $F_{n+1}$ of complete flags, i.e. the case $P=B$, where $B$ is the Borel subgroup of uppertriangular matrices.

Remark. After the first version of the paper was written the author discussed the topic with Vl. Zakalyukin, who later proved the following stronger result (conjected by the author). 
Proposition. For any pair of convex curves $\gamma_{1}: S^{1} \rightarrow \mathbb{P}^{n}$ and $\gamma_{2}: S^{1} \rightarrow \mathbb{P}^{n}$ the pairs $\left(\mathbb{P}^{n}, D_{\gamma_{1}}\right)$ and $\left(\mathbb{P}^{n}, D_{\gamma_{2}}\right)$ are diffeomorphic.

The main idea of his proof is to show the equivalence of the standard generating functions for the Legendre submanifolds which are lifts of the standard discriminants in the space of tangent elements $\mathbb{P} T^{*} \mathbb{P}^{n}$. Unfortunately this method does not give the topological part $b$ ) of the above main proposition.

Notice that in a sufficiently small neighborhood of any point $p$ of a locally convex curve $\gamma \subset \mathbb{P}^{n}$ its discriminant $D_{\gamma}$ is diffeomorphic to the standard discriminant $D i s c_{n}$, i.e. the set of all monic degree $n$ polynomials in one variable with real coefficients which have at least one real multiple root; see [Ish]. (In singularity theory $D i s c_{n}$ is also called the swallowtail.) Thus, in spite of the fact that $D_{\gamma}$ is highly singular, it has no local moduli. Still the existence of a global diffeomorphism of different $D_{\gamma}$ 's is quite a nontrivial fact.

The main motivation of this paper was an attempt to formalize the idea that any 2 convex curves are qualitatively equivalent in any natural sense. It is difficult to overestimate the role of my visit to the Max-Planck Institute during the summer of 1996, where the main bulk of this project was carried out. Stimulating discussions with M. Shapiro and Vl. Zakalyukin are highly appreciated.

\section{$\S 1$. ProOFs}

\section{Some generalities on convex curves.}

Definition. For any $t \in S^{1}$ and $1 \leq k \leq n-1$ let $L_{t}^{k}$ denote the osculating subspace to $\gamma$ at $\gamma(t)$ of dimension $k$.

1.1. Theorem (criterion of convexity). A curve $\gamma: S^{1} \rightarrow \mathbb{P}^{n}$ is convex if and only if for any $r$-tuple of positive integers $k_{1}, \ldots, k_{r}$ such that $\sum k_{i}=n$ and any $r$-tuple of pairwise different moments $t_{1}, \ldots, t_{r}$ the intersection $L_{t_{1}}^{n-k_{1}} \cap \ldots \cap L_{t_{r}}^{n-k_{r}}$ is a point.

Proof. In order to save space we refer the interested reader to [Co] and the references given there.

Definition. Given a nondegenerate curve $\gamma: S^{1} \rightarrow \mathbb{P}^{n}$, we define its dual $\gamma: S^{1} \rightarrow$ $\left(\mathbb{P}^{n}\right)^{*}$ to be the curve consisting of all osculating hyperplanes to $\gamma$.

Remark. If $\gamma$ is convex then $\gamma^{*}$ is also convex, see [Ar1], [Ar2].

Notation. If a point $p$ lies on some osculating hyperplane $H_{\tau}$ to $\gamma$, we say that the order of tangency $\sharp_{p}(\gamma(\tau))$ of $p$ at $\gamma(\tau)$ equals to $i$ if $p$ belongs to the osculating subspaces at $\gamma(\tau)$ of codimension at most $i$. (For example, for every point $p$ on a line $l$ tangent to a circle $c$ at $c(1)$ on $\mathbb{P}^{2}$ except for the tangency point $c(1)$ one has $\sharp_{p}(c(1))=1$. On the other side, $\sharp_{c(1)}(c(1))=2$.)

Given a nondegenerate $\gamma: S^{1} \rightarrow \mathbb{P}^{n}$ and a point $p \in \mathbb{P}^{n}$, we define the number of roots $\sharp_{p}(\gamma)$ of $p$ to be the sum of the orders of tangency $\sharp_{p}\left(\gamma\left(t_{i}\right)\right)$ taken over all osculating hyperplanes $H_{t_{i}}$ through $p$.

(The term 'number of roots' comes from the example when $\gamma$ is a rational normal curve in $\mathbb{P}^{n}$. In this case all points in $\mathbb{P}^{n}$ can be interpreted as homogeneous polynomials in 2 variables of degree $n$ with real coefficients (considered up to a constant factor), and $\gamma$ is the family of polynomials of the form $\left(a x_{1}+b x_{2}\right)^{n}, a^{2}+$ $b^{2} \neq 0$. In this situation $\sharp_{p}(\gamma)$ coincides with the total number of real roots of such a polynomial on $\mathbb{P}^{1}$, counted with multiplicities.) 
Observation. The number of roots $\sharp_{p}(\gamma)$ coincides with the total multiplicity (i.e. sum of local multiplicities) of the intersection of $\tilde{H}_{p}$ with $\gamma^{*}$. Here $\tilde{H}_{p}$ denotes the hyperplane in $\left(\mathbb{P}^{n}\right)^{*}$ corresponding to the point $p \in \mathbb{P}^{n}$.

1.2. Corollary. A curve $\gamma$ is convex if and only if for any $p \in \mathbb{P}^{n}$ one has $\sharp_{p}(\gamma) \leq$ $n$.

Projection. Given a convex curve $\gamma: S^{1} \rightarrow \mathbb{P}^{n}$ and its osculating hyperplane $H_{\tau}$ at the point $\gamma(\tau)$, let us denote by $\gamma^{\tau}: S^{1} \rightarrow H_{\tau}$ the curve obtained by projection of $\gamma$ onto $H_{\tau}$ along the pencil of tangent lines to $\gamma$, i.e. for any $t \in S^{1}$ one has $\gamma^{\tau}(t)=H_{\tau} \cap l_{t}$, where $l_{t}$ is the tangent line to $\gamma$ at $\gamma(t)$.

1.3. Lemma. For any $\tau \in S^{1}$ the curve $\gamma^{\tau}$ is a convex curve in $H_{\tau}$. Osculating hyperplanes to $\gamma^{\tau}$ and its discriminant $D_{\gamma^{\tau}}$ are obtained by intersection of the osculating hyperplanes and $D_{\gamma}$ with $H_{\tau}$.

Proof. The argument splits into 2 principal parts. First we show that $\gamma^{\tau}$ is nondegenerate, i.e. $\left(\gamma^{\tau}\right)^{\prime}(t), \ldots,\left(\gamma^{\tau}\right)^{(n-1)}(t)$ are linearly independent at any $t \in S^{1}$. Then we prove that $\gamma^{\tau}$ is convex, i.e. its total multiplicity of intersection with any hyperplane in $H_{\tau}$ does not exceed $n-1$. Observe that $\gamma$ has only one intersection point with $H_{\tau}$, namely $\gamma(\tau)$. Assume first that $t \neq \tau$. In this case one can choose a system of affine coordinates $x_{1}, \ldots, x_{n}$ in $\mathbb{P}^{n}$ such that $H_{\tau}$ coincides with the hyperplane $\left\{x_{n}=0\right\} ; \gamma(t)$ is the point with coordinates $(0, \ldots, 0,1)$ and the tangent line $l_{t}$ to $\gamma$ at $\gamma(t)$ is the $x_{n}$-axis. In these coordinates the curve $\gamma^{\tau}$ has the form

$$
\gamma^{\tau}(t)=\gamma(t)-\frac{\gamma_{n}(t)}{\gamma_{n}^{\prime}(t)} \gamma^{\prime}(t)
$$

where $\gamma_{n}$ is the last coordinate of $\gamma$. (Under our assumptions $\gamma_{n}^{\prime}(t) \neq 0$.) Therefore

$$
\left(\gamma^{\tau}\right)^{(i)}(t)=(-1)^{i} \frac{\gamma_{n}(t)}{\gamma_{n}^{\prime}(t)} \gamma^{(i)}(t)+\ldots,
$$

where ... denotes terms containing derivatives of $\gamma$ of order lower than $i$. By the above assumptions $\frac{\gamma_{n}(t)}{\gamma_{n}^{\prime}(t)} \neq 0$, and since $\gamma^{\prime}(t), \ldots, \gamma^{(n)}(t)$, are linearly independent one gets that the derivatives $\left(\gamma^{\tau}\right)^{(i)}(t), i=1, \ldots, n-1$, are linearly independent as well.

The alternative geometric argument is as follows. Since the point $\gamma(t)$ does not lie on $H^{\tau}$, one has that the osculating complete flag $f(t)$ to $\gamma$ at $\gamma(t)$ is transversal to $H_{\tau}$ and the same holds for all $t^{\prime}$ close to $t$. Therefore the complete flags obtained by intersection of $f\left(t^{\prime}\right)$ with $H_{\tau}$ are well defined. But in their turn these flags coincide with the osculating flags to $\gamma^{\tau}$, which are therefore well-defined in some neighborhood of $t$.

It remains to show that $\gamma^{\tau}$ is nondegenerate at $t=\tau$. This follows from the local calculation given below. In this case we can choose a system of coordinates such that in the neighborhood of $\tau$ (we assume $\tau=0$ ) the curve $\gamma$ has the form

$$
x_{1}=t+\ldots, x_{2}=t^{2}+\ldots, \ldots, x_{n}=t^{n}+\ldots .
$$


The osculating hyperplane $H^{0}$ at $\tau=0$ is given by $\left\{x_{n}=0\right\}$. The projected curve $\gamma^{0}(t)$ is given by

$$
\begin{aligned}
\gamma^{0}(t)=\gamma(t)-\frac{\gamma_{n}(t)}{\gamma_{n}^{\prime}(t)} \gamma^{\prime}= & \left(t+\ldots, t^{2}+\ldots, \ldots, t^{n}+\ldots\right) \\
& -\frac{t^{n}+\ldots}{n t^{n-1}+\ldots}\left(1+\ldots, 2 t+\ldots, \ldots, n t^{(n-1)}+\ldots\right) \\
= & \frac{1}{n}\left((n-1) t+\ldots,(n-2) t^{2}+\ldots, \ldots, t^{(n-1)}+\ldots, 0\right),
\end{aligned}
$$

which shows that $\gamma^{0}$ is nondegenerate at $t=0$.

Now we show that $\gamma^{\tau}$ is convex. By Corollary 1.2. one has to prove that for any $p \in H_{\tau}$ the number of roots $\sharp_{p}\left(\gamma^{\tau}\right)$ is less than or equal to $n-1$. This follows from the equality

$$
\sharp_{p}\left(\gamma^{\tau}\right)+1=\sharp_{p}(\gamma),
$$

which together with convexity of $\gamma$ gives the required result. Indeed, assume that some $p \in H_{\tau}$ lies in the intersection $L_{t_{1}}^{k_{1}}(\gamma) \cap L_{t_{2}}^{k_{2}}(\gamma) \cap \ldots \cap L_{t_{r}}^{k_{r}}(\gamma)$, where $t_{1}=\tau$. Since each subspace $L_{t_{i}}^{k_{i}}$ for $i \neq 1$ is transversal to $H_{\tau}$ (see the criterion of convexity), one has that $p$ lies in the intersection $L_{t_{1}}^{k_{1}}\left(\gamma^{\tau}\right) \cap L_{t_{2}}^{k_{2}-1}\left(\gamma^{\tau}\right) \cap \ldots \cap L_{t_{r}}^{k_{r}-1}\left(\gamma^{\tau}\right)$. Therefore, by definition of the number of roots, one gets the above equality.

For any $k$-tuple of moments $\left(t_{1}, \ldots, t_{k}\right), t_{i} \in S^{1}$, let $H_{t_{1}} \cap \ldots \cap H_{t_{k}}$ denote the intersection of the osculating hyperplanes $H_{t_{i}}, i=1, \ldots, k$. In what follows we use the following convention. If some of the moments $t_{j_{1}}, t_{j_{2}}, \ldots, t_{j_{r}}$ coincide we define the intersection $H_{t_{j_{1}}} \cap H_{t_{j_{2}}} \cap \ldots \cap H_{t_{j_{r}}}$ as the osculating subspace to $\gamma$ at $\gamma\left(t_{j_{1}}\right)$ of codimension $r$. Under this convention one has that $H_{t_{1}} \cap \ldots \cap H_{t_{k}}$ always has codimension $k$; see 1.1 .

1.4. Corollary. The projection $\gamma^{t_{1}, \ldots, t_{k}}$ of $\gamma$ onto any intersection of osculating hyperplanes $H_{t_{1}} \cap \ldots \cap H_{t_{k}}$ by a pencil of $k$-dimensional osculating subspaces to $\gamma$ is a convex curve. For any point $p \in H_{t_{1}} \cap \ldots \cap H_{t_{k}}$ one has

$$
\sharp_{p}\left(\gamma^{t_{1}, \ldots, t_{k}}\right)+k=\sharp_{p}(\gamma) .
$$

Proof. Apply the above lemma several times.

Elliptic hull of $\gamma$ and root filtration of $\mathbb{P}^{n}$.

Definition. For a convex $\gamma: S^{1} \rightarrow \mathbb{P}^{n}$ we define its elliptic hull Ell $\gamma$ to be the set of all $p \in \mathbb{P}^{n}$ with

$$
\left\{\begin{array}{l}
\sharp_{p}(\gamma)=0, \text { if } n \text { is even, } \\
\sharp_{p}(\gamma)=1, \text { if } n \text { is odd. }
\end{array}\right.
$$

1.5. Lemma. a) If $n$ is even then Ell ${ }_{\gamma}$ is a nonempty convex set in some affine chart of $\mathbb{P}^{n}$ (compare $[\mathrm{ShS}]$ ).

b) If $n$ is odd then Ell $l_{\gamma}$ is a disjoint union of $\bigcup_{\tau \in S^{1}}$ Ell $l_{\gamma^{\tau}}$ and, therefore, is fibered over $\gamma$ with a contractible fiber.

Proof. a) Note that if $\gamma: S^{1} \rightarrow \mathbb{P}^{2 k}$ is convex then $\gamma$ lies in some affine chart in $\mathbb{P}^{2 k}$. Indeed, take some osculating hyperplane $H_{\tau}$. The curve $\gamma$ is tangent to $H_{\tau}$ only at $\gamma(\tau)$ with the multiplicity $2 k$. Locally $\gamma$ lies on one side w.r.t. $H_{\tau}$. Therefore, one can make a small shift of $H_{\tau}$ in order to get rid of the intersection points with $\gamma$ 
near $\gamma(\tau)$. But no new intersection can appear for a sufficiently small shift, since the only intersection point of $\gamma$ and $H_{\tau}$ is $\gamma(\tau)$.

Assume now that $\mathbb{R}^{2 k} \subset \mathbb{P}^{2 k}$ is the affine chart containing $\gamma$. We claim that Ell coincides with the intersection $\bigcap_{\tau \in S^{1}} H_{a l f} f_{\tau}$. Here $\operatorname{Half}_{\tau}$ is the open halfspace in $\mathbb{R}^{2 k}$ containing $\gamma$ and bounded by the osculating hyperplane $H_{\tau}$. First of all, $\bigcap_{\tau \in S^{1}} H_{a l f} f_{\tau}$ is nonempty since it is an open convex set containing the interior of the convex hull of $\gamma$ in $\mathbb{R}^{2 k}$. Then $\bigcap_{\tau \in S^{1}} \mathrm{Half}_{\tau}$ is contained in Ell $\mathrm{l}_{\gamma}$. Indeed, every hyperplane through a point $p \in \bigcap_{\tau \in S^{1}} H_{a l f}$ is transversal to any osculating hyperplane $H_{\tau}$, since $p \notin H_{\tau}$. On the other side, $E l l_{\gamma} \subseteq \bigcap_{\tau \in S^{1}} H a l f_{\tau}$. Indeed, for every $p \notin H a l f_{\tau}, \tau \in S^{1}$, there exists a hyperplane $L_{p}$ through $p$ not intersecting $\gamma$ at all. Take the affine chart $\mathbb{P}^{n} \backslash L_{p}$ containing $\gamma$ and some pencil $\mathcal{L}_{p}$ of 'parallel' hyperplanes through $p$. Since $\gamma$ is a closed curve in $\mathbb{P}^{n} \backslash L_{p}$, one gets that some hyperplane in $\mathcal{L}_{p}$ does not intersect $\gamma$. Therefore, there exists a hyperplane in $\mathcal{L}_{p}$ tangent to $\gamma$ at some $\gamma\left(t_{p}\right)$. But this exactly means that the osculating hyperplane $H_{t_{p}}$ contains $p$.

b) Take a 1-parameter family of osculating hyperplanes. According to the proof of lemma 1.3, for any $\tau \in S^{1}$ the curve $\gamma^{\tau}$ is convex in $H_{\tau}$ and one has $\sharp_{p}\left(\gamma^{\tau}\right)+1=$ $\sharp_{p}(\gamma)$. Therefore the elliptic domain $E l l_{\gamma^{\tau}}$ of every curve $\gamma^{\tau}$ belongs to $E l l_{\gamma}$, i.e. $\bigcup_{\tau \in S^{1}} E l l_{\gamma^{\tau}} \subset E l l_{\gamma}$. (Note that the union $\bigcup_{\tau \in S^{1}} E l l_{\gamma^{\tau}}$ is disjoint.) Conversely, by definition, for odd $n$ every point $p$ in $E l l_{\gamma}$ has exactly one tangent hyperplane to $\gamma$, and thus $p$ belongs exactly to one osculating $H_{\tau}$. By the equality $\sharp_{p}\left(\gamma^{\tau}\right)+1=\sharp_{p}(\gamma)$, the point $p$ lies in the elliptic hull of $\gamma^{\tau}$. Moreover, by the first part of this proof, $E l l_{\gamma^{\tau}}$ is a convex domain in $H_{\tau}$ and, therefore, is contractible, which gives the necessary result.

Definition. By the root filtration of $\mathbb{P}^{n}$ w.r.t. a convex curve $\gamma: S^{1} \rightarrow \mathbb{P}^{n}$,

$$
\mathbb{P}_{0}(\gamma) \subset \ldots \subset \mathbb{P}_{\left[\frac{n}{2}\right]}(\gamma)=\mathbb{P}^{n},
$$

we mean the filtration where each $\mathbb{P}_{i}(\gamma)$ consists of all $p \in \mathbb{P}^{n}$ for which the number of roots $\sharp_{p}(\gamma)$ is greater than or equal to $n-2 i$.

Let $\mathcal{T}^{j}=\left(S^{1}\right)^{j}$ denote the $j$-dimensional torus and let $\mathcal{T}^{j} / \mathfrak{S}_{j}$ be its quotient modulo the natural action of the symmetric group $\mathfrak{S}_{j}$ by permutation of copies of $S^{1}$.

1.6. Lemma. a) For any $n$ and $0 \leq i \leq\left[\frac{n}{2}\right]$ the set $\mathbb{P}_{i}(\gamma) \backslash \mathbb{P}_{i-1}(\gamma)$ is naturally fibered over $\mathcal{T}^{n-2 i} / \mathfrak{S}_{n-2 i}$ with a contractible fiber. (For $n=2 k$ and $i=k$ the set $\mathbb{P}_{k}(\gamma) \backslash \mathbb{P}_{k-1}(\gamma)$ is contractible; see 1.5.a.)

b) This fibration is trivial.

Proof. a) Every point $p \in \mathbb{P}_{i}(\gamma) \backslash \mathbb{P}_{i-1}(\gamma)$ can be described as follows. There exists a unique $(n-2 i)$-tuple of osculating hyperplanes $H_{t_{1}}, \ldots, H_{t_{n-2 i}}$ to $\gamma$ (with probably coinciding moments $t_{1}, \ldots, t_{n-2 i}$, in which case we use the same convention as above) such that $p$ belongs to the intersection $H_{t_{1}} \cap H_{t_{2}} \cap \ldots \cap H_{t_{n-2 i}}$ and, moreover, lies in the elliptic hull of the curve $\gamma^{t_{1}, \ldots, t_{n-2 i}}$. (Here $\gamma^{t_{1}, \ldots, t_{n-2 i}}$ is the projection of $\gamma$ onto $H_{t_{1}} \cap H_{t_{2}} \cap \ldots \cap H_{t_{n-2 i}}$ by the pencil of osculating subspaces of dimension $n-2 i$.) Indeed, we have that $\sharp_{p}\left(\gamma^{t_{1}, \ldots, t_{n-2 i}}\right)+2 i=\sharp_{p}(\gamma)$; see 1.2. Therefore $p$ must lie in the elliptic hull of $\gamma^{t_{1}, \ldots, t_{n-2 i}}$. On the other hand, any intersection $H_{t_{1}} \cap H_{t_{2}} \cap \ldots \cap H_{t_{n-2 i}}$ has codimension $n-2 i$ (see 1.1) and any curve $\gamma^{t_{1}, \ldots, t_{n-2 i}}$ is convex. Therefore, applying 1.5 , we get the necessary result. 
b) The fibration of elliptic components $E l l_{\gamma^{t_{1}}, \ldots, t_{n-2 i}}$ of the curves $\gamma^{t_{1}, \ldots, t_{n-2 i}}$ over the set of moments $\left(t_{1}, \ldots, t_{n-2 i}\right) \in \mathcal{T}^{n-2 i} / \mathfrak{S}_{n-2 i}$ depends continuously on $\gamma \in \mathfrak{C o n}_{n}$. Since $\mathfrak{C o n}_{n}$ consists of 1 connected component (up to orientation for odd $n)$ it suffices to show that the fibration sending $E l_{\gamma^{t_{1}, \ldots, t_{n-2 i}}}$ to $\left(t_{1}, \ldots, t_{n-2 i}\right)$ is trivial for some $\gamma \in \mathfrak{C o n}_{n}$.

The simplest example showing triviality is the case when $\gamma$ is a rational normal curve. Indeed, in this case the space under consideration is the fibration of the space $\Pi_{n}(i)$ all homogeneous forms of degree $n$ in 2 variables (up to a scalar multiple) which have exactly $n-2 i$ real zeros (counted with multiplicities) over the space $\mathcal{T}^{n-2 i} / \mathfrak{S}_{n-2 i}$ of their real zeros. But $\Pi_{n}(i)$ has the obvious structure of the product of the space of degree $n-2 i$ polynomials with all real zeros (considered up to a scalar multiple) and the space of degree $2 i$ polynomials with no real zeros (considered up to a scalar multiple). This shows that the fibration $\Pi_{n}(i) \rightarrow \mathcal{T}^{n-2 i} / \mathfrak{S}_{n-2 i}$ is trivial.

Proof of the main proposition. a) We will construct the homeomorphism of pairs $\left(\mathbb{P}^{n}, D_{\gamma_{1}}\right)$ and $\left(\mathbb{P}^{n}, D_{\gamma_{2}}\right)$ in $\left[\frac{n}{2}\right]+1$ steps. On the $i$ th step, $i=0, \ldots,\left[\frac{n}{2}\right]$, we obtain the partial homeomorphism $h_{i}$ of the terms $\mathbb{P}_{i}\left(\gamma_{1}\right)$ and $\mathbb{P}_{i}\left(\gamma_{2}\right)$ of the above filtration.

The initial step. We construct the homeomorphism $h_{0}: \mathbb{P}_{0}\left(\gamma_{1}\right) \rightarrow \mathbb{P}_{0}\left(\gamma_{2}\right)$. Indeed, each of $\mathbb{P}_{0}\left(\gamma_{1}\right)$ and $\mathbb{P}_{0}\left(\gamma_{2}\right)$ is homeomorphic to $\mathcal{T}^{n} / \mathfrak{S}_{n}$ as follows. Every element in $\mathcal{T}^{n} / \mathfrak{S}_{n}$ is a pair $\left(t_{1}, \ldots, t_{r}\right) \in\left(\mathcal{T}^{r} \backslash \operatorname{Diag}\right) / \mathfrak{S}_{r}, r \leq n$, and $\left(k_{1}, \ldots, k_{r}\right), \sum k_{i}=$ $n$. We map such a pair $\left(t_{1}, \ldots, t_{r}\right),\left(k_{1}, \ldots, k_{r}\right)$ onto the intersection point $L_{t_{1}}^{n-k_{1}}\left(\gamma_{j}\right) \cap$ $\ldots \cap L_{t_{r}}^{n-k_{r}}\left(\gamma_{j}\right), j=1,2$. This identification provides the homeomorphism $h_{0}$ : $\mathbb{P}_{0}\left(\gamma_{1}\right) \rightarrow \mathbb{P}_{0}\left(\gamma_{2}\right)$ by 1.1 .

The typical step. Each point in $\mathbb{P}_{i}\left(\gamma_{j}\right) \backslash \mathbb{P}_{i-1}\left(\gamma_{j}\right)$ lies in the elliptic hull of the unique curve $\gamma^{t_{1}, \ldots, t_{n-2 i}} \subset H_{t_{1}} \cap \ldots \cap H_{t_{n-2 i}}$, i.e. the set of (not necessarily pairwise different) moments $\left(t_{1}, \ldots, t_{n-2 i}\right) \in \mathcal{T}^{n-2 i} / \mathfrak{S}_{n-2 i}$ is uniquely defined. For each individual intersection $H_{t_{1}} \cap \ldots \cap H_{t_{n-2 i}}$ the homeomorphism $h_{i-1}$ is already defined on the complement to the elliptic hulls of the curves $\gamma_{1}^{t_{1}, \ldots, t_{n-2 i}}$ and $\gamma_{2}^{t_{1}, \ldots, t_{n-2 i}}$. Since the elliptic hulls are convex domains and the fibrations of the elliptic hulls over $\mathcal{T}^{n-2 i} / \mathfrak{S}_{n-2 i}$ are trivial, we can extend $h_{i-1}$ fiberwise to $h_{i}$ by identifying the points of the elliptic hull of $\gamma_{1}^{t_{1}, \ldots, t_{n-2 i}}$ with points of the elliptic hull of $\gamma_{2}^{t_{1}, \ldots, t_{n-2 i}}$ for all tuples $\left(t_{1}, \ldots, t_{n-2 i}\right) \in \mathcal{T}^{n-2 i} / \mathfrak{S}_{n-2 i}$

b) The corresponding component Comp of $^{n} \backslash D_{\gamma}$ contained in $\mathbb{P}_{i} \backslash \mathbb{P}_{i-1}$ is fibered over $\left(\mathcal{T}^{n-2 i} \backslash\right.$ Diag $) / \mathfrak{S}_{n-2 i}$ with the contractible fiber. Since in fact $\left(\mathcal{T}^{n-2 i} \backslash\right.$ Diag $) / \mathfrak{S}_{n-2 i}$ is contractible to $S^{1}$ for any $n-2 i>0$, one gets that $C o m p_{i}$ is contractible to $S^{1}$ for all $n$ and $i \leq\left[\frac{n}{2}\right]$, except for $E l l_{\gamma}$ for even $n$, which is contractible to a point.

Remark. For convex algebraic curves the above homeomorphism (constructed rather explicitly) can be made (piecewise) real algebraic. This leads to the interesting problem of studying properties of the complexification of this homeomorphism in the case when the initial convex curves are not projectively equivalent.

\section{REFERENCES}

[Ar1] V. I. Arnold, On the number of flattening points on space curves, preprint of the MittagLeffler Institute (1) (1994/95), 1-13, Sinai's Moscow seminar on Dynamical Systems, AMS Transl., Ser. 2, vol. 171, 1995, pp. 11-22. MR 96i:53070 
[Ar2] V. I. Arnold, Topological problems in the theory of wave propagation, Russian Math. Surveys 51 (1) (1996), 1-49. MR 97c:58001

[Co] W. A. Coppel, Discojugacy, Lecture Notes in Math., vol. 220, Springer-Verlag, 1971. MR 57:778

[Ish] G. Ishikawa, Developable of a curve and determinacy relative to osculating type, Quart. J. Math., Oxford Ser (2) 46 (184) (1995), 437-451. CMP 96:06

[Sh1] B. Shapiro, Space of linear differential equations and flag manifolds, Math. USSR - Izv. 36 (1) (1990), 183-197. MR 91g:58030

[Sh2] B. Shapiro, Towards qualitative theory for high order linear ODE, in preparation.

[ShS] B. Shapiro and V. Sedykh, On Young hulls of convex curve in $\mathbb{R}^{2 n}$, preprint MPI 96-95.

[MSh] M. Shapiro, Topology of the space of nondegenerate curves, Math. USSR - Izv. 57 (1993), 106-126. MR 94k:58019

Department of Mathematics, University of Stockholm, S-10691, Sweden

E-mail address: shapiro@matematik.su.se 